\title{
Les stades ailés de Baetis samochai Koch 1981 du Proche- Orient (Insecta : Ephemeroptera)
}

\author{
Z. Moubayed1
}

Les imagos et subimagos or et $\%$ de Baetis samochai Koch, 1981 sont décrites sur du matériel en provenance de la plaine de la Békaa (Liban).

The adult stages of Baetis samochai Koch, 1981 from the near east (Insecta : Ephemeroptera).

Imagines and subimagines $\sigma$ and $q$ of Baetis sa mochai Koch, 1981 are described from material collected from the plain of the Bekaa in Lebanon.

L'étude de la faune benthique de deux réseaux hydrographiques du Liban (plaine de la Békaa) a permis la récolte des stades imaginal et subimaginal, encore inconnus, de Bactis samochai Koch, 1981. En voici la description, à partir de materiel conservé en alcool à $70^{\circ}$.

\section{1. - Imago or}

Longueur de l'aile antérieure : 4,6 à 5,5 mm en mai, 6,1 mm en mars.

Tête : Yeux en turban rouge brique clair peu convexes du côté interne, à contour peu divergent et presque subrectiligne. Région latéro-externe inférieure des yeux bleue noirâtre. Face et antennes brunes; ocelles clairs.

Thorax: Brun foncé brillant, en particulier le mésonotum. Pattes grisâtres, les antérieures gris brunâtre. Ailes hyalines; sur les antérieures, nervation de l'aire ptérostigmatiques très variable (fig. 1-1); sur les postérieures, un processus costal bien développé et trois nervures longitudinales dont la seconde n'est pas ramifiée (fig. 1-2).

\footnotetext{
1. Laboratoire d'Hydrobiologie (ERA 702), Université Paul Sabatier, 118, route de Narbonne, 31062 Toulouse Cedex.

Ce travail fait partie d'un mémoire de thèse en préparation en vue de l'obtention du Doctorat d'Etat.
}

Abdomen : Dans l'ensemble, brun terne un peu grisâtre. Tergites de couleur unie, sternites plus clairs avec, jusqu'à l'avant dernier inclus, des maculations syrmétriques brun plus foncé : deux « accents circonflexes" antérieurs et deux points postérieurs.

Neuvième sternite clair au centre, avec seulement les deux points et des tâches brunes le long des pleures. Cerques brun grisâtre uni assez foncé, à jointures claires.

Genitalia : (fig. 1-3). Coxites clairs avec une tâche brun foncé à la base. Gonopodes nettement arqués, à article terminal court et arrondi. Bord apical interne des coxites nettement roulé et, de ce fait, proéminent mais par contre dépourvu de processus tronqué. Second segment des gonopodes non armé d'une pointe interne.

La larve de $B$, samochai a été rangée par Koch (1981) dans le groupe vermus, mais les caractères des genitalia éloignent plutôt ces deux espèces (voir Müller-Liebenau, 1969 et Kimmins, 1972).

\section{2. - Imago ?}

Ensemble à peu près entièrement brun grisâtre moyen. Bords du mésonotum et sclérites pleuraux plus foncés. Nervation alaire plus épaisse et brun plus foncé que chez le $\sigma$. Cerques grisâtres, de plus en plus clairs vers l'apex. 


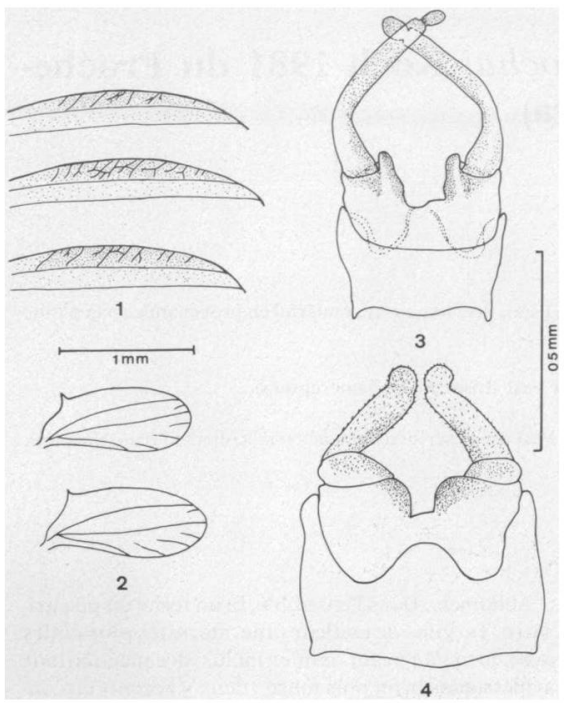

Fig. 1. Baetis samochi. Koch. 1 : aire ptérostigmatique de trois imagos mâles. 2 : aile postérieure de deux imagos mâles. 3 : genitalia de l'imago mâle, vue ventrale. 4 : id, subimago mâle.

\section{3. - Subimagos or et $\%$.}

Ailes gris terne uniforme ; thorax jaunâtre à maculations linéaires brunes sur les bords du mésonotum et sur les pleures, surtout en arrière du prothorax et au dessus de la troisième coxa. Abdomen grisâtre sale chez le $\sigma$, gris jaunatre plus clair chez la $Q$ à cause des oeufs. Génitalia ơ : fig : 1-4.

\section{4. - Matériel examiné}

144 imagos $1 \circ, 11$ imagos $९, 12$ subimagos $\circ$, $5 \mathrm{~s} Q$ et plusieurs dizaines de larves âgées provenant de 3 localités de la plaine de la Békaa (Liban) $)^{2}$ :

1. 5 imagos ơ sont déposées au Muséum de la Faculté des Sciences (Université Libanaise) de Beyrouth.

2. La situation géographique des localitès de récoltes est illus. trée sur une carte du Liban (Giani, Martinez-Ansemil et Moubayed 1982).
- Rivière Litani (Jib-Jennine), alt. $800 \mathrm{~m}$.

$10.5 .82: 43 \sigma, 4 \%, 2 \mathrm{~s} \sigma, 1 \mathrm{~s} \&$.

$16.5 .82: 79 \sigma, 6 \mathrm{~s} \circ, 2 \mathrm{~s}$.

- Marécages d'Ammik, alt. $850 \mathrm{~m}$.

29.3.82: $3 \sigma, 1$ \&, $1 \mathrm{~s}$.

$16.5 .82: 10 \sigma, 5 \%, 1 \mathrm{~s}$ \%

- Yammouné, alt. $1300 \mathrm{~m}$.

23.5.82: 9 o*, 1 \&, $3 \mathrm{~s}$ o, $1 \mathrm{~s}$ \%.

L'espèce a été déterminée par examen de larves et d'exuvies larvaires au dernier stade, récoltées dans les mêmes prélèvements que les adultes.

\section{5. - Ecologie sommaire}

Au Liban, B. samochai n'avait été récoltée jusqu'à présent que dans deux localités situées sur des affluents du Litani, à Zahlé et à Chtaura (Koch 1981). Dans la plaine de la Békaa, nous l'avons récoltée aux états adulte et larvaire. Tant à Yammouné (NordEst) qu'à Ammik ou à Jib-Jennine (Litani), l'espèce vit en eau calme mais renouvelée ou en courant lent (10 v $25 \mathrm{~cm} / \mathrm{s})$.

Son abondance dans la rivière Litani montre qu'elle colonise bien les milieux riches en macrophytes (Ranunculus, Myriophyllum, Sparganium etc...) et en algues filamenteuses. Les substrats fins, limoneux à limono-sableux, et chargés en matière organique constituent un habitat favorable à l'espèce qui apparaît aussi modérément thermophile $\left(t^{\circ} \operatorname{maxi}\right.$. male relevée : $23^{\circ} \mathrm{C}$, à Jib-Jennine). En outre, nous avons noté à cette station de fortes densités de zooplancton (Cladocères, Copépodes et Ostracodes).

\section{Remerciements}

Je remercie Mr A. Thomas qui a bien voulu confirmer la détermination de l'espèce, relire et critiquer le manuscrit.

Travaux eltés

Giani (N.), Martinez-Ansemil (E.) et Moubayed (Z.). 1982. - Les oligochètes aquatiques du Liban. I1. Premier inventaire faunistique. Annls. Limnol., 18 (3) : 7-13.

Kimmins (D. E.). 1954. - A revised key to the adults of Ephemeroptera with notes on their ecology. Scient. Publs. Freshwat. biol. Ass., 2 nd edition 1972, $\mathrm{N}^{\circ} 15: 1-75$.

Koch (S.). 1981. - Baetis samochai $\mathrm{n}$. sp. aus dem vorderen Orient (Ephemeroptera : Baetidae). Ent. Z mil insekt. 91 (11): 121-125.

Muller-Liebenau (I.). 1969. - Revision der europaichen Arten der Gattung Baetis Leach, 1815 (Insecta, Ephemeroptera). Gewäss. Abwäss 48/49:1-214, 